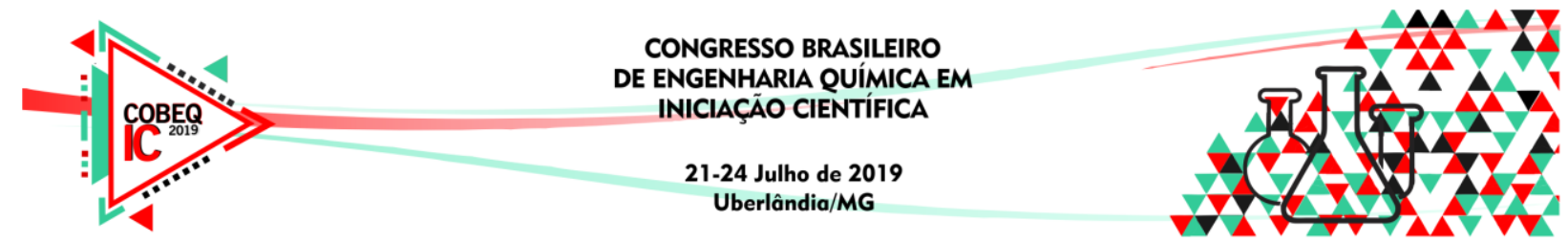

\title{
AVALIAÇÃO DA QUALIDADE DE ÁGUA DE CONSUMO EM COMUNIDADE VULNERÁVEL NO ESTADO DE SERGIPE
}

\author{
P. C. BORTOLUZZI ${ }^{1}$, R. A. PRATA ${ }^{1}$, T. C. BRITO ${ }^{1}$, A. B. HORA ${ }^{2}$ e M. N. MARQUES ${ }^{3}$ \\ ${ }^{1}$ Universidade Tiradentes, Faculdade de Engenharia Química \\ ${ }^{2}$ Universidade Tiradentes, Pós-graduação em Saúde e Ambiente \\ ${ }^{3}$ Instituto de Tecnologia e Pesquisa, Universidade Tiradentes, Pós-graduação em Saúde e \\ Ambiente \\ E-mail para contato: pamela.cunha@ souunit.com.br
}

\begin{abstract}
RESUMO - A universalização da água potável e segura em quantidade e qualidade suficientes, ainda se apresenta como um grande desafio, levando em consideração as desigualdades sociais e ambientais, sobretudo nas regiões mais pobres, e grupos mais vulneráveis. Desta forma, assegurar o fornecimento de água de qualidade para os diversos grupos sociais, dentre eles as comunidades quilombolas, deve ser uma ação de efetiva realização para prevenção de doenças de contaminação hídrica como previsto na Portaria $n^{\circ}$ 5/2017 do Ministério da Saúde. Objetiva-se dessa forma avaliar a qualidade da água para o consumo humano na Comunidade Quilombola de Pontal da Barra - Barra dos Coqueiros, Sergipe. Após o reconhecimento de área, foram definidos pontos para coleta de água, sendo estas realizadas de acordo com os Standard Methods for the Examination of Water and Wastewater (APHA, 1998). Dentre os resultados, a maioria dos parâmetros físico-químicos e químicos encontram-se de acordo com os valores permissíveis da Portaria $n^{\circ}$ 5/2017, apresentando variações de condutividade sem riscos à saúde. Porém, os parâmetros microbiológicos apresentaram presença de coliformes. Com isso os resultados demonstraram a eficiência no tratamento da água, entretanto, a manipulação e armazenamento realizado pela população se mostrou ineficiente quanto a manutenção da qualidade da água, o que pode prejudicar a saúde da comunidade local.
\end{abstract}

\section{INTRODUÇÃO}

A água é um recurso essencial para a vida, com isso, torna-se necessário sua distribuição em quantidade suficiente e qualidade adequada. Sua importância em uma comunidade consiste na promoção de hábitos higiênicos e na prevenção de doenças. Porém, esse abastecimento não ocorre devidamente, visto que uma parcela considerável da população mundial não tem acesso direto a esse bem (FERREIRA et al., 2014).

Em 2016, de acordo com o Sistema Nacional de Informações sobre Saneamento (BRASIL, 2018), o maior índice de atendimento com rede de distribuição de água se localizava na região sudeste com cerca de $91,2 \%$, enquanto regiões como norte e nordeste apresentavam os índices mais baixos com $55,4 \%$ e $73,6 \%$, respectivamente. 


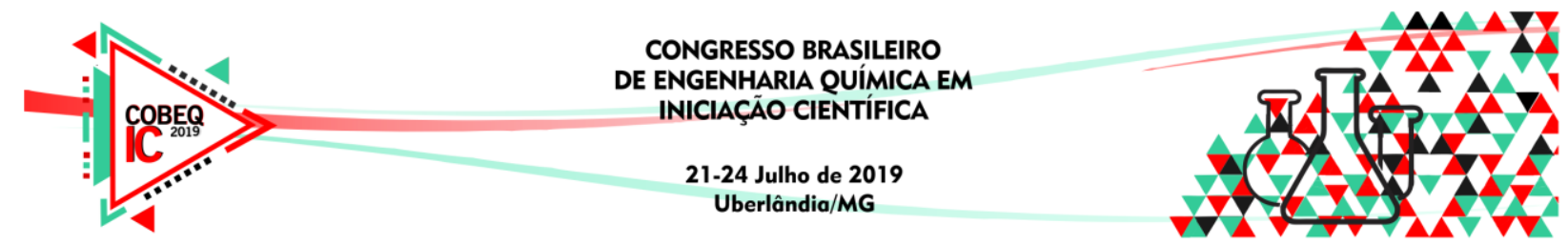

Quando a distribuição de água não ocorre pelo abastecimento público, ela é suprida por meios subterrâneos, muito presentes em comunidades vulneráveis, principalmente nas comunidades quilombolas existentes em todo país especialmente, no Nordeste onde se concentram 63\% delas (BRASIL, 2013).

Por conta da sua vulnerabilidade, é necessário monitorar a água utilizada por essas comunidades para garantir o padrão estabelecido pelas legislações, a partir dos parâmetros previamente dispostos pela Portaria $n^{\circ}$ 5/2017 do Ministério da Saúde (BRASIL, 2017) e pelas Resoluções do Conselho Nacional do Meio Ambiente (CONAMA) no 396/2008 (BRASIL, 2008). Por meio desses parâmetros, é possível monitorar a água e sua qualidade que associado a fatores de saúde é capaz de demonstrar o nível de qualidade da água de uma comunidade, estando esse proporcionalmente ligado a qualidade de vida da população.

Portanto, este trabalho tem por objetivo: Avaliar a qualidade de água para o consumo humano na comunidade quilombola da Pontal da Barra, localizada no município de Barra dos Coqueiros, no estado de Sergipe, afim de analisar parâmetros físico-químicos, microbiológicos e químicos,

\section{MATERIAIS E MÉTODOS}

\section{1. Área de estudo}

A área de estudo é a comunidade de Pontal da Barra, localizada no município de Barra dos Coqueiros no estado de Sergipe, como apresentado na Figura 1. A escolha da comunidade de Pontal da Barra deve-se por se tratar de uma comunidade quilombola, na qual parte da população mesmo com a distribuição de água tratada, ainda faz uso de água subterrânea.

Após o reconhecimento de área, foram definidos os pontos para a realização da coleta de água para os testes físico-químicos e as análises microbiológicas e químicas, sendo esse processo realizado entre novembro de 2017 e maio de 2019. As descrições dos pontos de coleta estão apresentadas na Tabela 1.

Tabela 1 - Identificação e localização dos pontos na comunidade

\begin{tabular}{llll}
\hline Ponto & Descrição & Classificação & Coordenadas UTM \\
\hline P1 & Distribuição & Tratada & $\mathrm{X}=0733835$ Y $=8811142$ \\
P2 & Poço & Água subterrânea (bruta) & $\mathrm{X}=0742378$ Y $=8819928$ \\
\hline
\end{tabular}

As análises físico-químicas foram realizadas in situ, e posteriormente, as amostras foram coletadas, acondicionadas em recipientes de vidro e, conservadas em temperatura próxima de $4^{\circ} \mathrm{C}$ de acordo com o Standard Methods for the Examination of Water and Wastewater (SMEWW) 1060 e o Guia Nacional de Coleta e Preservação de Amostras da Agência Nacional de Águas (ANA), preservando-as assim, até a realização dos processos analíticos microbiológicos e químicos. 


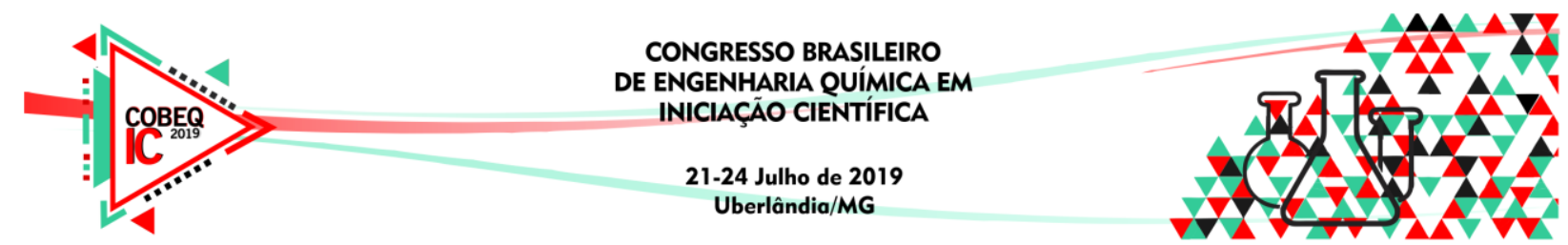

Figura 1 - Comunidade Pontal da Barra (Barra dos Coqueiros - SE).

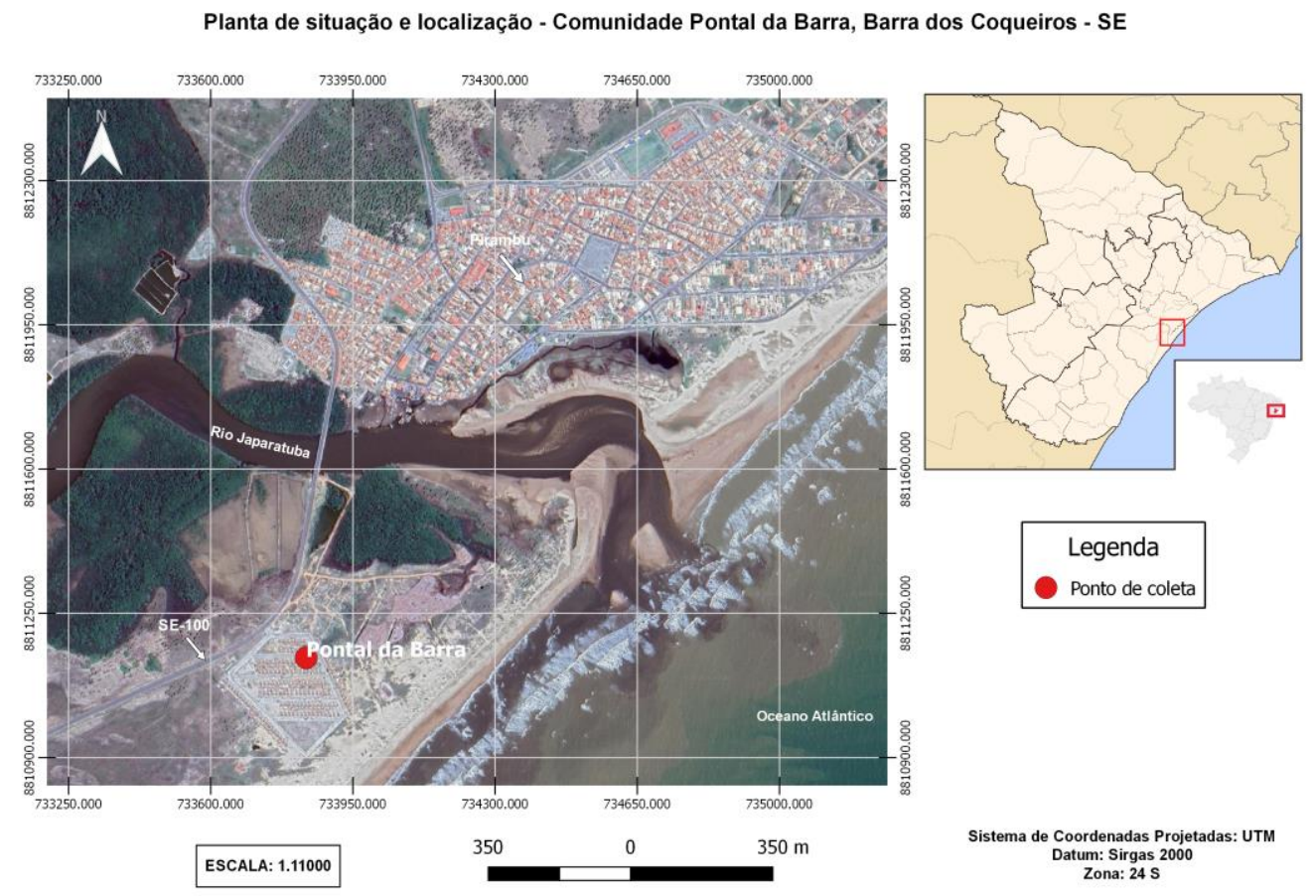

Fonte: Adaptado do mapa de Raphael Lorenzeto de Abreu, 2006.

\subsection{Determinação dos parâmetros físico-químicos e químicos da água}

As análises físico-químicas foram realizadas por meio da sonda multiparâmetros da Hanna Instrument, modelo HI 9828. Este equipamento foi utilizado para medição de temperatura, $\mathrm{pH}$, oxigênio dissolvido (OD), condutividade, sólidos dissolvidos totais (SDT), e salinidade. Destaca-se, que antes das análises era realizada a calibração do equipamento com solução padrão. Já as análises químicas, foram realizadas com o medidor multiparâmetro à prova d'água - Micro 20 da Akso, onde foram medidos os parâmetros de cloro livre, cloro total, nitrito, nitrato, sulfato, amônia e turbidez.

\subsection{Determinação dos parâmetros microbiológicos da água}

Os métodos escolhidos para as análises microbiológicas foram baseados no SMEWW 9000, onde cada metodologia aplicada para cada ensaio microbiológico, está descrita na Tabela 2.

Tabela 2 - Metodologia adotada para as análises microbiológicas de qualidade de água

\begin{tabular}{cll}
\hline Ensaio & Método & Unidade \\
\hline Coliformes totais & SMEWW 9221 A & NMP.100 mL-1 \\
Coliformes termotolerantes & SMEWW 9221 B & NMP.100 mL-1 \\
\hline
\end{tabular}




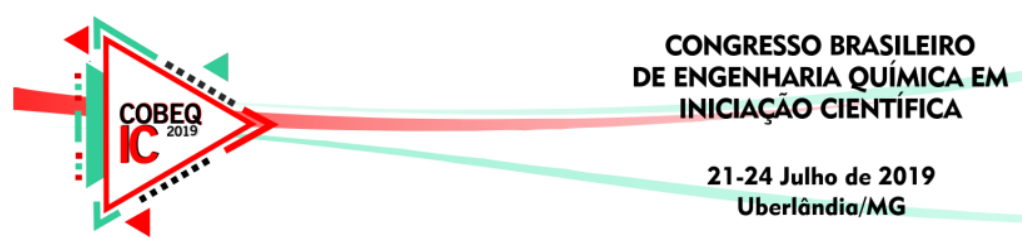

\section{RESULTADOS E DISCUSSÃO}

Os resultados físico-químicos, microbiológicos e químicos obtidos das amostras estão presentes nas tabelas 3, 4 e 5, respectivamente.

Tabela 3 - Estatísticas de máximo, mínimo, média e desvio dos parâmetros físico-químicos

\begin{tabular}{|c|c|c|c|c|c|c|c|}
\hline ڤે & & $\begin{array}{c}\text { Temperatura } \\
\text { da Água }\left({ }^{\circ} \mathrm{C}\right) \\
\text { Indif. }\end{array}$ & $\begin{array}{c}\mathbf{p H} \\
6,0 \text { a } 8,5\end{array}$ & $\begin{array}{c}\text { OD } \\
(\mathrm{mg} / \mathrm{L})\end{array}$ & $\begin{array}{c}\text { Condutividade } \\
(\mu \mathrm{S} / \mathrm{cm})\end{array}$ & $\begin{array}{c}\text { SDT } \\
(\mathbf{m g} / \mathbf{L}) \\
<1000\end{array}$ & $\begin{array}{c}\text { Salinidade } \\
(\mathbf{m g} / \mathbf{L}) \\
<200\end{array}$ \\
\hline \multirow{4}{*}{$\bar{a}$} & Mínimo & 27,50 & 5,96 & 1,70 & 185 & 88 & 80 \\
\hline & Máximo & 31,70 & 7,30 & 9,90 & 734 & 367 & 350 \\
\hline & Média & 29,89 & 6,55 & 5,58 & 384 & 191 & 180 \\
\hline & DP & 1,55 & 0,52 & 3,10 & 206 & 104 & 101 \\
\hline \multirow{4}{*}{$\Sigma$} & Mínimo & 25,16 & 6,25 & 1,55 & 148 & 74 & 70 \\
\hline & Máximo & 31,44 & 7,22 & 7,35 & 630 & 315 & 300 \\
\hline & Média & 29,80 & 6,74 & 3,64 & 388 & 193 & 184 \\
\hline & DP & 2,61 & 0,41 & 2,42 & 233 & 116 & 113 \\
\hline
\end{tabular}

$\mathrm{DP}=$ desvio padrão

Tabela 4 - Estatísticas de máximo, mínimo, média e desvio dos parâmetros microbiológicos

\begin{tabular}{cccc}
\hline Pontos & & $\begin{array}{c}\text { Coli. Totais (NMP/100 mL) } \\
\text { Ausência em } 100 \mathrm{~mL}\end{array}$ & $\begin{array}{c}\text { Termotolerantes (NMP/100 mL) } \\
\text { Ausência em } 100 \mathrm{~mL}\end{array}$ \\
\hline \multirow{4}{*}{ P1 } & NHC & $<1$ \\
& Mínimo & 62 & 7,3 \\
& Máximo & 26 & 2,6 \\
& Média & 25 & 3,6 \\
\hline \multirow{4}{*}{ P2 } & DP & NHC & $<1$ \\
& Mánimo & 33 & 7,8 \\
& Média & 11 & 3,0 \\
& DP & 14 & 4,1 \\
\hline
\end{tabular}

$\mathrm{DP}=$ desvio padrão

Tabela 5 - Resultados das análises químicas

\begin{tabular}{lcc}
\multicolumn{1}{c}{ Análise } & P10219 & P20219 \\
\hline Cloro livre (mg/L) & 0,03 & 0,05 \\
Cloro total (mg/L) & 0,09 & 0,07 \\
Nitrito (mg/L) & 0 & 0 \\
Nitrato (mg/L) & $<0,12$ & $<, 12$ \\
Sulfato (mg/L) & 17 & 66 \\
Amônia (mg/L) & 0 & 0,59 \\
Turbidez (NTU) & $<7$ & $\mathbf{1 0}$ \\
\hline
\end{tabular}

Analisando os resultados físico-químicos (Tabela 3), observa-se que o $\mathrm{pH}$ do ponto P1 $(5,96)$ da coleta de fevereiro/2018, está abaixo do valor mínimo permitido pela portaria de potabilidade $n^{\circ}$ 5/2017 do Ministério da Saúde. A Tabela 3 também demonstra que os valores 


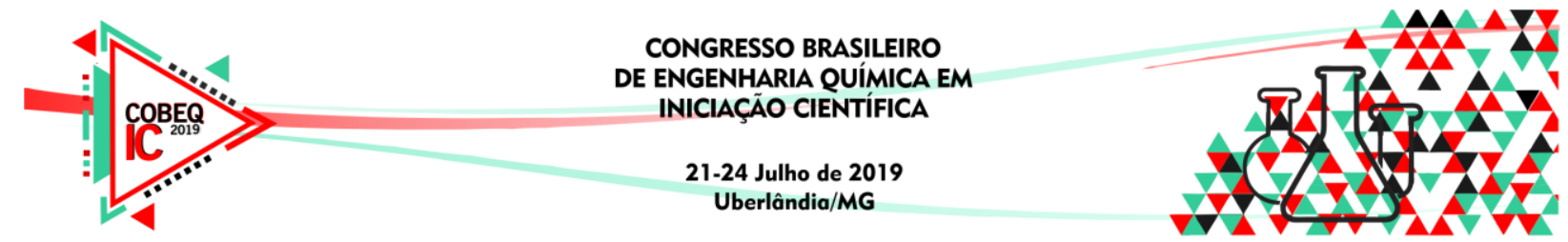

de condutividade estão acima de $100 \mu \mathrm{S} / \mathrm{mL}$, de acordo com o Guia Nacional de Coleta e Preservação de Amostras da ANA (2011), as concentrações de condutividade acima de 100 $\mu \mathrm{S} / \mathrm{mL}$ podem servir como indicador de características corrosivas da água e ambientes impactados.

As análises químicas realizadas na última coleta como controle final, demonstraram somente a turbidez do ponto $\mathrm{P} 2$ acima dos valores máximos permissíveis pela portaria $\mathrm{n}^{\circ}$ 5/2017 do Ministério da Saúde, porém ainda sem demonstrar grandes riscos à saúde.

Em relação aos parâmetros microbiológicos, observa-se um nível preocupante de coliformes termotolerantes nas coletas realizadas em dezembro/2018 e fevereiro/2019 para os pontos P1 com 5,5 e 7,3 NMP/100 mL, e P2 com 7,8 e 7,2 NMP/100 mL, respectivamente.

Os coliformes totais incluem bactérias na forma de bastonetes gram-negativos, anaeróbios facultativos, capazes de fermentar a lactose com produção de gás. Atualmente sabe-se que existem pelo menos três gêneros, Escherichia, Enterobacter e Klebsiella. A Escherichia coli faz parte da família de coliformes termotolerantes e é considerado um dos micro-organismos mais comuns no corpo humano, principalmente no trato digestório. Esse patógeno produz enterotoxinas que causam a diarreia, mal-estar e cólicas abdominais (PONGELUPPE et al., 2009; MORAIS, et al., 2016; FRANCO, LANDGRAF, 2008).

\section{CONCLUSÃO}

1. Os parâmetros físico-químicos e químicos, em sua maioria, estão de acordo com os valores máximos permissíveis da Portaria $n^{\circ}$ 5/2017 do Ministério da Saúde, e CONAMA no 396/2008 sem grandes riscos à saúde;

2. Condutividade relativamente alta para água de abastecimento nas amostragens realizadas em ambos os pontos $\mathrm{P} 1$ e $\mathrm{P} 2$, com valores acima de $100 \mu \mathrm{S} / \mathrm{mL}$;

3. Presença de coliformes termotolerantes em dezembro/2018 e fevereiro/2019 para os pontos P1 e P2 (média final de 2,6 e 3,0 NMP/100 mL, respectivamente), apresentando riscos à saúde;

4. Em geral, a água tratada distribuída para a comunidade é de boa qualidade, indicando assim, a eficiência do tratamento da mesma. Apresentando, porém, crescimento de coliformes nas últimas coletas realizadas, sendo um fator de risco para os moradores da comunidade.

\section{AGRADECIMENTOS}

Fundação de Apoio à Pesquisa e a Inovação Tecnológica do Estado de Sergipe (Fapitec), Coordenação de Aperfeiçoamento de Pessoal de Nível Superior (Capes), Comitê da Bacia do Rio Japaratuba, Programa Pesquisa para o SUS (PPSUS), Programa de Bolsas de Iniciação Científica (PROBIC/Unit), Universidade Tiradentes (Unit), Instituto de Tecnologia e Pesquisa (ITP). 


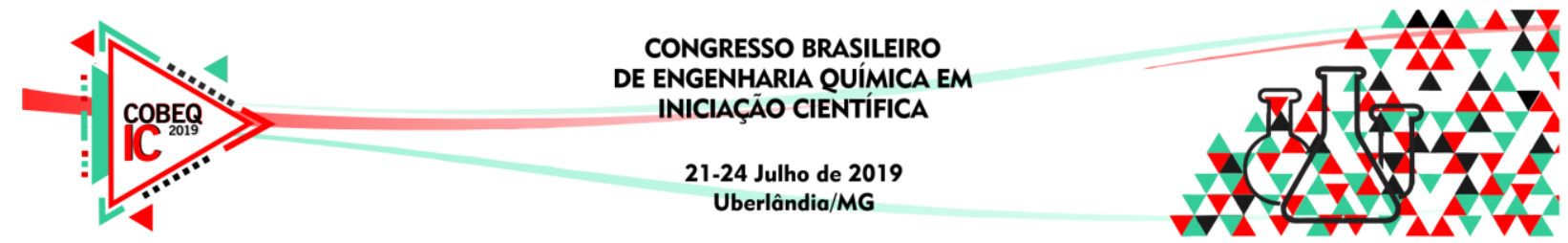

\section{REFERÊNCIAS}

ABREU, R. L.

Lista de mesorregiões e microrregiões de Sergipe. Wikipédia. Disponível em: <https://pt.wikipedia.org/wiki/Lista_de_mesorregi\%C3\%B5es_e_microrregi\%C3\%B5 es_de_Sergipe>. Acesso em: 5 maio 2019.

ANA - Agência Nacional de Água; CETESB - Companhia Ambiental do Estado de São Paulo.

Guia Nacional de Coleta e Preservação de Amostras. Água, sedimento, comunidade aquáticas e efluentes líquidos. Brasília-DF, 2011.

APHA - American Public Health Association.

Standard Methods for the Examination of Water and Wastewater. Americam Water Works Association, Water Environmental Federation, 20 ${ }^{\text {th }}$ ed. Washington, 1998.

BRASIL, Conselho Nacional do Meio Ambiente (CONAMA).

Resolução $n^{\circ} 396$. Dispõe sobre a classificação e diretrizes ambientais para o enquadramento das águas subterrâneas e dá outras providências. Publicado no D.O.U n ${ }^{\circ} 66$, abril-2008.

BRASIL. Guia de Políticas Públicas para Comunidades Quilombolas - Programa Brasil Quilombola.

Secretaria de Políticas de Promoção da Igualdade Racial. Secretaria de Políticas para Comunidades Tradicionais, 2013.

BRASIL. Ministério das Cidades. Secretaria Nacional de Saneamento Ambiental - SNSA.

Sistema Nacional de Informações sobre Saneamento: Diagnóstico dos Serviços de Água e Esgotos - 2016. Brasília: SNSA/MCIDADES, 2018. 220 p.: il.

BRASIL. Ministério da Saúde.

Portaria de Consolidação $n^{\circ}$ 5, de 28 de setembro de 2017. Anexo XX, Origem: PRT MS/GM 2914/2011. Brasília, DF, set. 2017.

FERREIRA, E. P.; FERREIRA, J. T. P.; PANTALEÃO, F. S.; FERREIRA, Y. P.; ALBUQUERQUE, K. N. A.; FERREIRA, T. C.

Abastecimento de Água para Consumo Humano em Comunidades Quilombolas no Município de Santana do Mundaú - Al. Revista Brasileira de Geografia Física, vol.07, n.06 (2014) 1119-1125.

FRANCO, B. D. G. M.; LANDGRAF, M.

Microbiologia dos Alimentos. São Paulo: Editora Atheneu, 2008.

PONGElUPPE, A. T.; OlIVEIRA, D. B.; SILVA, E. A.; AGUILEIRA, K. K.; ZITEI, V.; BASTOS, M. F.

Avaliação de Coliformes Totais, Fecais e Enterobactérias em Bebedouros Localizados em uma Instituição de Ensino de Guarulhos. Revista Saúde-UNG-Ser, v. 3, n. 2, p. 5 9, 2009.

MORAIS, W. A.; SALEH, B. B.; ALVES, W. S.; AQUINO, D. S.

Qualidade sanitária da água distribuída para abastecimento público em Rio Verde,Goiás, Brasil. Cad. Saúde Colet., 2016, Rio de Janeiro, 24 (3): 361-367. 